\title{
Photon Correlation Spectroscopy as a Witness for Quantum Coherence
}

\author{
Carlos Sánchez Muñoz® and Frank Schlawin® \\ Clarendon Laboratory, University of Oxford, Parks Road, Oxford OX1 3PU, United Kingdom
}

(Received 10 January 2020; accepted 28 April 2020; published 18 May 2020)

\begin{abstract}
The development of spectroscopic techniques able to detect and verify quantum coherence is a goal of increasing importance given the rapid progress of new quantum technologies, the advances in the field of quantum thermodynamics, and the emergence of new questions in chemistry and biology regarding the possible relevance of quantum coherence in biochemical processes. Ideally, these tools should be able to detect and verify the presence of quantum coherence in both the transient dynamics and the steady state of driven-dissipative systems, such as light-harvesting complexes driven by thermal photons in natural conditions. This requirement poses a challenge for standard laser spectroscopy methods. Here, we propose photon correlation measurements as a new tool to analyze quantum dynamics in molecular aggregates in driven-dissipative situations. We show that the photon correlation statistics of the light emitted in several models of molecular aggregates can signal the presence of coherent dynamics. Deviations from the counting statistics of independent emitters constitute a direct fingerprint of quantum coherence in the steady state. Furthermore, the analysis of frequency resolved photon correlations can signal the presence of coherent dynamics even in the absence of steady state coherence, providing direct spectroscopic access to the much sought-after site energies in molecular aggregates.
\end{abstract}

DOI: 10.1103/PhysRevLett.124.203601

Introduction.-The emerging field of quantum thermodynamics assesses the role of quantum fluctuations on thermodynamic properties of meso- or nanoscopic systems [1-4]. Of particular importance is the possible role of quantum coherence to enhance the efficiency of thermodynamic processes in the quantum regime and the possible gain for technological applications. It has stimulated a lot of theoretical investigations into the detection of coherence $[5,6]$, the circumstances when this advantage can be gained [7-10], and how much work can be gained from a quantum system [11-13]. In parallel, recent years have also seen an intense debate in the field of chemical physics. Sparked by the discovery of quantum-mechanical coherence in photosynthetic complexes, it was hypothesized that coherent dynamics following the absorption of sunlight might be relevant for the functionality of these complexes, and even form a key ingredient for the high quantum efficiency of solar light harvesting [14-16].

However, as these discoveries are based on phasecoherent ultrafast laser experiments [17], it was argued that transient coherences should be irrelevant for natural photosynthesis, and they rather constitute an artefact of the laser usage $[18,19]$. Accordingly, natural photosynthesis instead takes place in a nonequilibrium steady state, and only coherence in such steady state regimes could reasonably be expected to have functional relevance. A growing number of theoretical studies discusses the impact of light statistics on the ensuing dynamics [20-24]. In addition, a considerable number of theoretical models have been put forth, where steady state coherence is induced by environments [7,25-32], both relating to photosynthesis and chemical reactions more generally. Yet to date, there exists no direct spectroscopic probe of these nonequilibrium steady states. The detection of transient coherence in ultrafast laser spectroscopy, too, relies on a comparison to model calculations, in order to infer the presence or absence of coherence [33,34].

In this Letter, we introduce photon correlation spectroscopy as a possible tool to measure coherent dynamics and the presence of steady state coherence between excitons in single molecule spectroscopy. Our proposal relies on measurements of photon correlations of the light emitted by single molecular aggregates [35-40] in natural, nonequilibrium situations. In particular, we show how the presence of steady-state coherence can be detected in two-photon coincidence measurements by varying the detection polarization. We further prove that frequency-resolved counting statistics [41-47] can reveal similar information on transient coherent dynamics as two-dimensional spectroscopy $[48,49]$. The analysis of these signals could provide access to the local onsite energies in molecular aggregates, which form one of the greatest unknowns in theoretical models of such complexes [50-52]. Crucially, this information is obtained in a nonequilibrium stationary state, without the need of phase-coherent ultrafast pulses. As photon correlation spectroscopy considers properties of the fluorescence emitted from a sample, it can be carried out with excitation by incoherent light sources, and thus provide insights into nonequilibrium dynamics in conditions of natural illumination of the samples. 
Nonlinear laser spectroscopy vs photon correlation measurements. - On a microscopic level, nonlinear spectroscopy measures the nonlinear response functions of a sample, which can be connected to dipole correlation functions [17]. For instance, standard techniques such as pump-probe measurements or photon echoes measure the third-order nonlinear susceptibility. Ultrashort pulses prepare a nonequilibrium state, whose evolution is monitored by a probe pulse after fixed time delay. This amounts to a measurement of response functions of the form $\sim\left\langle\hat{d}_{i}\left(t_{3}\right)\left[\hat{d}_{j}\left(t_{2}\right),\left[\hat{d}_{k}\left(t_{1}\right),\left[\hat{d}_{l}(0), \varrho_{0}\right]\right]\right]\right\rangle$, where $d_{i}$ denote components of the sample's dipole operator. Phase matching and control of the laser polarizations allow for additional selectivity, in order to only measure quantum pathways with the desired information [53,54], distinguish homogeneous from inhomogeneous broadening, or detect bath correlations from two-dimensional resonance line shapes [48]. Multicolor extensions of such measurements further enable the detection of correlations between electronic and vibrational dynamics [55].

As we will see below, the conceptually simpler measurement of photon coincidences, a central quantity in quantum optics described by Glauber's second-order correlation function $g^{(2)}\left(t_{1}, t_{2}\right)$, contains similar information, as the fluorescence light can be directly translated into a function of the sample dipole operator. Measurements of bunching and antibunching statistics are widely employed to characterize quantum effects in continuously driven cavity quantum electrodynamics systems [56,57], and are recently gaining relevance in other fields, e.g., as a method to achieve super-resolution in biological imaging [58]. Its most general version measures coincidences between photons detected at different energywindows [41-47], described by the frequency-filtered Glauber's second-order correlation function [41]:

$g^{(2)}\left(\omega_{1}, t_{1} ; \omega_{2}, t_{2}\right)=\frac{\left\langle: \mathcal{T}\left[\prod_{i=1}^{2} \hat{E}_{\omega_{i}, \Gamma}^{(-)}\left(t_{i}\right) \hat{E}_{\omega_{i}, \Gamma}^{(+)}\left(t_{i}\right)\right]:\right\rangle}{\prod_{i=1}^{2}\left\langle\hat{E}_{\omega_{i}, \Gamma}^{(-)}\left(t_{i}\right) \hat{E}_{\omega_{i}, \Gamma}^{(+)}\left(t_{i}\right)\right\rangle}$,

(a)

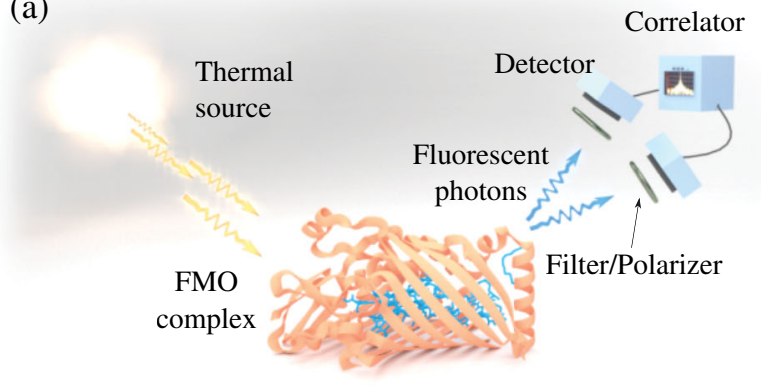

where $\mathcal{T}(:)$ refers to time (normal) ordering, and with $\hat{E}_{\omega_{i}, \Gamma}^{( \pm)}(t)$ the negative/positive frequency parts of the timedependent electric field operator filtered at the frequency $\omega$ by a Lorentzian filter of linewidth $\Gamma, \hat{E}_{\omega, \Gamma}(t)=$ $(\Gamma / 2) \int_{0}^{\infty} \exp \left[-(i \omega+\Gamma / 2) t^{\prime}\right] \hat{E}\left(t-t^{\prime}\right) \mathrm{d} t^{\prime}$. Here, we will be concerned with the simplest case of zero-delay statistics in the stationary regime, $g^{(2)}\left(\omega_{1}, \omega_{2}\right) \equiv$ $\lim _{t \rightarrow \infty} g^{(2)}\left(\omega_{1}, t ; \omega_{2}, t\right)$. Even in the zero-delay case, the frequency-filtered electric field involves an integral in time, therefore containing information not only about the stationary values of the density matrix $\rho$, but also about the dynamics of the system, formally encoded in the Liouvillian superoperator $\mathcal{L}$ that generates the evolution of the density matrix, $\dot{\rho}=\mathcal{L} \rho$ [59]. From Eq. (1) and the definition of $\hat{E}_{\omega, \Gamma}(t)$, it is clear that photon correlation measurements too can be traced back to four-point correlation functions of the sample dipole operators, which is directly proportional to the radiated electric field (see below). Therefore, as pointed out in Refs. [60,61], they can provide similar spectroscopic information as the measurement of the third-order nonlinear response using ultrafast laser pulses, with the important advantage that, rather than using ultrashort pulses to initiate the dynamics, they can be performed in a steady state configuration.

Emission statistics from a single dimer.-Now, we demonstrate the use of photon counting statistics to identify coherence in the simplest conceivable composite quantum system: a dimer composed of two dipoles described in the two-level system (TLS) approximation [see Fig. 1(b)]. It forms the simplest toy model to describe the formation of excitonic states in molecular aggregates and their signatures in ultrafast spectroscopy. As a consequence, dimer systems have been widely studied in both theory [62-66] and experiments [67], in order to better understand dynamic or spectroscopic features in more complex realistic models of light-harvesting complexes.

Each dipole has a dipolar moment operator $\hat{\mathbf{d}}_{i}=$ $\mu_{i}\left(\hat{\sigma}_{i}+\hat{\sigma}_{i}^{\dagger}\right) \mathbf{u}_{i}$, where $\hat{\sigma}_{i}$ is the lowering operator of the $i$ th TLS and $\mathbf{u}_{i}$ are unit vectors. The energy structure of this

FIG. 1. (a) Sketch of the idea of photon-counting spectroscopy. Information about the state of a system (which might well be a stationary density matrix) is inferred through the statistics of photon-counting events. (b) Geometry of the dimer-detector system. (c) Bare basis of our toy model, Eq. (3). (d) Excitonic basis which diagonalizes the dipole Hamiltonian, Eq. (3). 
system is shown in Fig. 1(c), with $|d\rangle \equiv \hat{\sigma}_{1}^{\dagger} \hat{\sigma}_{2}^{\dagger}|g g\rangle$ denoting the doubly excited state. Considering the bare energies of the TLSs to be $\omega_{\sigma}$, and that any internal coupling rate is $g \ll \omega_{\sigma}$, the positive-frequency part of far-zone electric field operator radiated by the dimer is given by $\hat{\mathbf{E}}^{(+)}(\mathbf{r}, t)=$ $\mathbf{E}_{1} \hat{\sigma}_{1}(t-|\boldsymbol{r}| / c)+\mathbf{E}_{2} \hat{\sigma}_{2}(t-|\boldsymbol{r}| / c) \quad$ [68], with $\mathbf{E}_{i} \equiv$ $E_{r} \mu_{i} \sin \eta_{i} \mathbf{x}_{\mathbf{i}}$ and $E_{r} \equiv \omega_{\sigma}^{2} / 4 \pi \epsilon_{0} c^{2}|\mathbf{r}|$. This turns Eq. (1) into a four-point dipole correlation function. The angles $\eta_{i}$ and the unit vectors $\mathbf{x}_{\mathbf{i}}$ are defined in Fig. 1(b). In the following, we consider that the detectors measure a specific polarization $\mathbf{u}_{\lambda}$, yielding scalar fields $\hat{E}^{(+)}(\mathbf{r}, t)=$ $E_{1, \lambda} \hat{\sigma}_{1}(t-|\boldsymbol{r}| / c)+E_{2, \lambda} \hat{\sigma}_{2}(t-|\boldsymbol{r}| / c)$, where $\mathbf{E}_{1(2), \lambda} \equiv E_{1(2)}$. $\mathbf{u}_{\lambda}$. Using the angle definitions shown in Fig. 1(b), and omitting from now on the subscript $\lambda$ for convenience, we can write $E_{i}=E_{r} \mu_{i} \sin \eta_{i} \cos \phi_{i}$, with $\phi_{1}=\varphi, \phi_{2}=\theta-\varphi$. Thus, $E_{1}$ and $E_{2}$ are determined by the relative orientation of the dipoles $\theta$, the polarizer angle $\varphi$, and the position of the detector. To simplify the discussion, we first consider unfiltered photon counting statistics on a single detector, as sketched in Fig. 1(b). Thus, Glauber's zero-delay, second-order correlation function simply reads $g^{(2)}(0)=$ $\left\langle\hat{E}^{(-)} \hat{E}^{(-)} \hat{E}^{(+)} \hat{E}^{(+)}\right\rangle /\left\langle\hat{E}^{(-)} \hat{E}^{(+)}\right\rangle^{2}$. Writing the correlators in terms of the elements of the density matrix $\rho_{i j}=\langle i|\hat{\rho}| j\rangle$ as $\left\langle\hat{\sigma}_{1}^{\dagger} \hat{\sigma}_{2}^{\dagger} \hat{\sigma}_{1} \hat{\sigma}_{2}\right\rangle=\rho_{d d},\left\langle\hat{\sigma}_{1}^{\dagger} \hat{\sigma}_{1}\right\rangle=\rho_{e_{1} e_{1}}+\rho_{d d},\left\langle\hat{\sigma}_{2}^{\dagger} \hat{\sigma}_{2}\right\rangle=\rho_{e_{2} e_{2}}+$ $\rho_{d d}$ and $\left\langle\hat{\sigma}_{1}^{\dagger} \hat{\sigma}_{2}\right\rangle=\rho_{e_{2} e_{1}}$, we straightforwardly obtain

$$
\begin{aligned}
g^{(2)}(0)= & 4\left(E_{1} E_{2}\right)^{2} \rho_{d d} /\left[E_{1}^{2} \rho_{e_{1} e_{1}}+E_{2}^{2} \rho_{e_{2} e_{2}}\right. \\
& \left.+\left(E_{1}^{2}+E_{2}^{2}\right) \rho_{d d}+2 E_{1} E_{2} \Re \rho_{e_{2} e_{1}}\right]^{2} .
\end{aligned}
$$

Bounded photon fluctuations in uncoupled systems.Importantly, Eq. (2) features the steady-state coherence $\rho_{e_{2} e_{1}}$ in the denominator. This terms enters the equation through $\left\langle\hat{E}^{(-)} \hat{E}^{(+)}\right\rangle$, which may suggest that a simple measurement of the intensity could suffice to determine the existence or absence of coherence. However, the intensity alone cannot distinguish coherence without a priori knowledge of the dipole strength of the sample.

On the other hand, a measurement of $g^{(2)}(0)$ can unambiguously verify the presence of excitonic coherence in the steady state. To prove this, we will now evaluate Eq. (2) for a reference case in which no coherence exists. This reference case consists of two TLSs with no coherence, neither in the bare or the excitonic (energy) basis and no population imbalance, i.e., a state that fulfils the following three no-coherence conditions: (i) $\left\langle\hat{\sigma}_{i}\right\rangle=0$, (ii) $\left\langle\hat{\sigma}_{i}^{\dagger} \hat{\sigma}_{i}\right\rangle=$ constant, and (iii) $\left\langle\hat{O}_{i} \hat{O}_{j}\right\rangle=\left\langle\hat{O}_{i}\right\rangle\left\langle\hat{O}_{j}\right\rangle$, where $\hat{O}_{i}$ is any operator of the $i$ th emitter and $i \neq j$. This is the expected situation, for instance, in the case of excitation by sunlight. In terms of the density matrix elements, these conditions read (i) $\rho_{e_{1} e_{2}}=\rho_{e_{2} e_{1}}=0$, (ii) $\rho_{e_{1} e_{1}}=\rho_{e_{2} e_{2}} \equiv p$, and (iii) $\rho_{d d}=p^{2}(1+p)^{2}$. We now show that photon correlations allow us to identify states where these nocoherence conditions are not fulfilled, therefore signalling the presence of coherence. Substituting these conditions into Eq. (2), we find $g^{(2)}(0)=4\left(E_{1} E_{2}\right)^{2} /\left(E_{1}^{2}+E_{2}^{2}\right)^{2}$. Defining the ratio $r=E_{2} / E_{1}$, we get $g^{(2)}(0)=4 r^{2} /$ $\left(1+r^{2}\right)^{2}$, which has an upper bound of 1 . This yields the restriction $g^{(2)}(0) \leq 1$ for uncoupled emitters, allowing to define the criterion $g^{(2)}(0)>1$ to identify coherence. We carry out a similar analysis for an aggregate of $N$ emitters in the Supplemental Material [69], showing that, in the most general case in which $N$ is unknown, we can establish a more general criterion $g^{(2)}(0)>2$ to signal coherence.

Specific model.-We now provide a particular example on how coherence can be detected by fulfilling these inequalities in the case of a dimer. To this end, we introduce a driven-dissipative dynamical model of the dimers in which we will tune the steady state coherence by introducing ad hoc asymmetric incoherent pumping.

The model consists of two coupled TLSs with frequencies $\omega_{\sigma} \pm \Delta / 2$, described by the dipole Hamiltonian

$$
\begin{aligned}
\hat{H}_{d}= & \left(\omega_{\sigma}-\Delta / 2\right) \hat{\sigma}_{1}^{\dagger} \hat{\sigma}_{1}+\left(\omega_{\sigma}+\Delta / 2\right) \hat{\sigma}_{2}^{\dagger} \hat{\sigma}_{2} \\
& +J\left(\hat{\sigma}_{1}^{\dagger} \hat{\sigma}_{2}+\text { H.c. }\right),
\end{aligned}
$$

where $J$ denotes coherent coupling between the TLSs. The eigenstates for $J=0$ correspond to the bare or site basis, $\left\{|g g\rangle,\left|e_{1}\right\rangle,\left|e_{2}\right\rangle,|d\rangle\right\}$, see Fig. 1(c). In the general case $J \neq 0$, the eigenstates in the one-excitation subspace are $|+\rangle=c\left|e_{1}\right\rangle+s\left|e_{2}\right\rangle$ and $|-\rangle=s\left|e_{1}\right\rangle-c\left|e_{2}\right\rangle$, where $c=1 / \sqrt{1+\xi^{-2}}, s=1 / \sqrt{1+\xi^{2}}, \xi=J /(\Delta / 2+R)$ and $R=\sqrt{J^{2}+(\Delta / 2)^{2}}$, so that the corresponding energies are $\omega_{ \pm}=\omega_{\sigma} \pm R$. We will consider the general case in which $R \neq 0$ and greater than the natural linewidths in the system, i.e., we will assume that one can observe two spectrally resolved peaks in the emission at the energies $\omega_{ \pm}$. In order to keep the peak separation fixed, regardless of its possible origin, we parametrize $\Delta$ and $J$ by a mixing angle $\beta$ as $J=R \cos \beta$ and $\Delta / 2=R \sin \beta$. Thus, the case $\beta=0$ corresponds to two peaks originating from two coupled, resonant dipoles, and $\beta=\pi / 2$ to two uncoupled, detuned dipoles.

The driven-dissipative dynamics of the dimers in the presence of losses and incoherent driving is described by the master equation $\dot{\hat{\rho}}=-i[\hat{H}, \hat{\rho}]+\sum_{i} \gamma \mathcal{D}_{\hat{\sigma}_{i}}\{\hat{\rho}\} / 2+$ $\sum_{i} P_{i} \mathcal{D}_{\hat{\sigma}_{i}^{\dagger}}\{\hat{\rho}\} / 2$, with $\mathcal{D}_{\hat{\mathcal{O}}}\{\hat{\rho}\} \equiv 2 \hat{\mathcal{O}} \hat{\rho} \hat{\mathcal{O}}^{\dagger}-\hat{\mathcal{O}}^{\dagger} \hat{\mathcal{O}} \hat{\rho}-\hat{\rho} \hat{\mathcal{O}}^{\dagger} \hat{\mathcal{O}}$, where $\gamma$ is the decay rate of both dimers and $P_{i}$ the incoherent pumping rate of the $i$ th dimer. Importantly, we allow for different incoherent excitation rates that can give rise to population imbalance. This is relevant since coherences in the excitonic basis are given by $\rho_{-+}=$ $c s\left(\rho_{e_{1} e_{1}}-\rho_{e_{2} e_{2}}\right)+s^{2} \rho_{e_{2} e_{1}}-c^{2} \rho_{e_{1} e_{2}}$. Thus, in order to have coherence in the excitonic basis we need coherence and/or population imbalance in the site basis, which we can enforce by setting $J \neq 0$ (so $c \neq 0$ ) and $P_{1} \neq P_{2}$. As shown in Fig. 2(a), the pumping imbalance gives rise to steady 


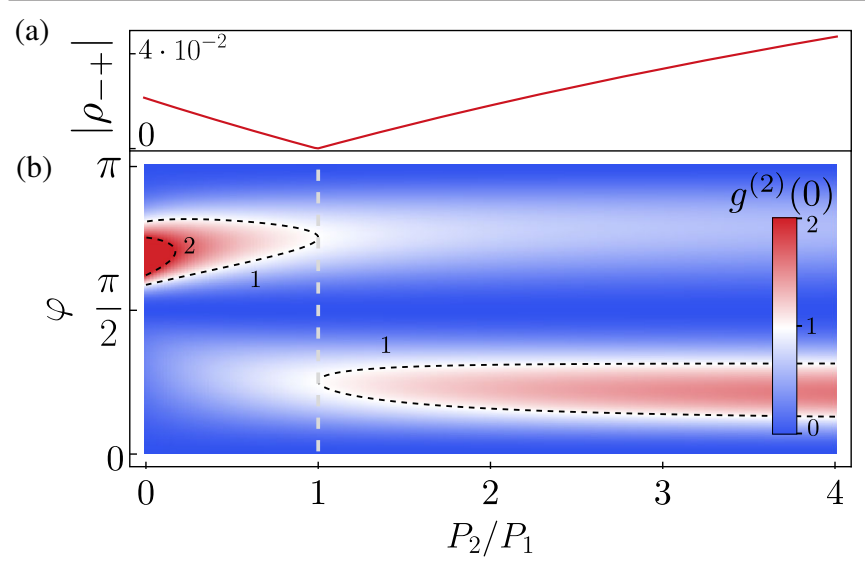

FIG. 2. (a) Coherence between excitonic states versus the pumping anisotropy $P_{2} / P_{1}$. When $P_{1}=P_{2}$, no excitonic coherence exists. (b) Dependence of $g^{(2)}(0)$ on the polarization angle $\varphi$ and the anisotropy $P_{2}$. Parameters: $R=4 \gamma, \beta=\pi / 3, J=2 \gamma$, $\theta=\pi / 2, P_{1}=0.1 \gamma$. Coherence is evidenced by the existence of regions with $g^{(2)}(0)>1$.

state coherence between the excitonic states of the dimer which is directly proportional to the asymmetry of the pumps [69]. This allows us to illustrate the potential of $g^{(2)}(0)$ measurements to unveil coherences in the excitonic basis, as we demonstrate in Fig. 2(b), where $g^{(2)}(0)$ is plotted vs the anisotropy of the pumping and the polarizer angle of the detector setup. This plot reveals regions with $g^{(2)}(0)>1$, hence signalling the presence of steady state coherence in a dimer, and even $g^{(2)}(0)>2$, which signals coherence in a system regardless of the number of emitters [69]. Only when there is coherence in the steady state, the variation of the polarizer angle yields a value larger than unity for some angle that depends on the dimer parameters [69], and thus enables the direct detection of steady state coherence. This is the first main result of our Letter, and is supported by further calculations for larger aggregates shown in the Supplemental Material [69]. Crucially, while the fulfilment of the criterion implies the existence of coherence, the reverse is not necessarily true [69], and therefore this measurement should be thought of as a coherence witness.

Frequency-resolved measurements.-The previous analysis has been devoted to revealing coherence in the stationary density matrix of the system using photon correlation measurements. Now, we explore how frequency-resolved measurements [see Eq. (1)] introduce a time resolution that allows us to reveal transient coherent dynamics even when these average to zero in the steady state [see Fig. 3(a)], allowing us to distinguish this case from that of detuned, uncoupled emitters. In our model, we can describe coherent dynamics that yield zero stationary coherences by setting $J \neq 0, P_{1}=P_{2}$, which is the case we consider from now on.

From now on, we will be concerned with the light emitted at the two possible transition energies between

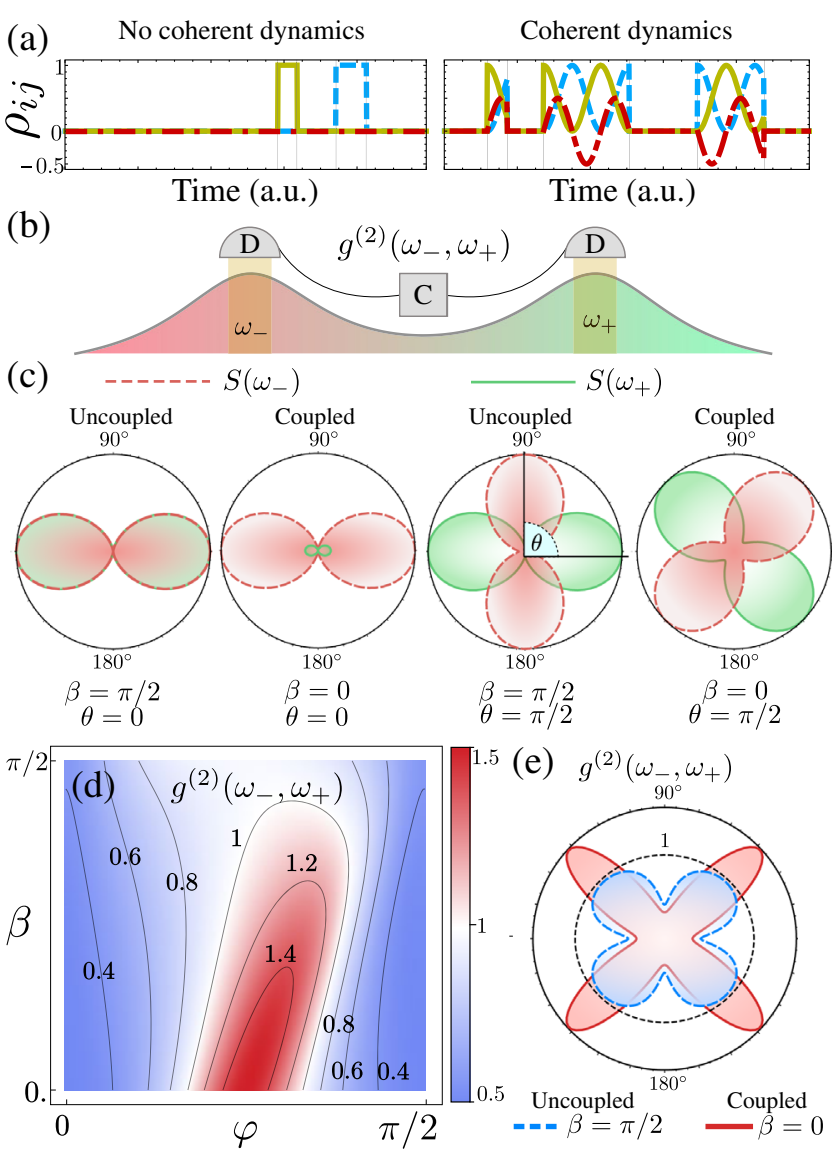

FIG. 3. (a) Monte Carlo trajectories depicting $\rho_{e_{1} e_{1}}$ (solid, yellow line), $\rho_{e_{2} e_{2}}$ (dashed, blue line), and $\rho_{e_{1} e_{2}}$ (dot-dashed, red line) during the process of absorbing and emitting several photons, with and without coherent couplings. For $P_{1}=P_{2}$, the oscillatory dynamics of the coherences average to zero steadystate coherence. (b) Sketch of a frequency-resolved correlation setup measuring correlations between the two spectral peaks at $\omega_{ \pm}$. (c) Polarization measurements of the spectrum of emission at frequencies $\omega_{ \pm}$for different values of coherent coupling $J=$ $R \cos \beta$ and interdipole angle $\theta$. (d),(e) Cross-correlation measurements at $\theta=\pi / 2$ versus detection polarization for different values of $\beta$. Parameters: $R=2 \gamma, P_{1}=P_{2}=0.1 \gamma, \Gamma=\gamma$.

eigenstates, $\omega_{ \pm}$, see Fig. 3(b). Figure 3(c) shows the polarization profile of the light emitted at these two frequencies, given by $S\left(\omega_{ \pm}\right)=\lim _{t \rightarrow \infty}\left\langle\hat{E}_{\omega_{ \pm}, \Gamma}^{(-)}(t) \hat{E}_{\omega_{ \pm}, \Gamma}^{(+)}(t)\right\rangle$, for several combinations of $\beta$ and $\theta$. If we consider that most of the emission will come from the single-excitation subspace, so that $S\left(\omega_{ \pm}\right) \propto\left|\left\langle g g\left|\hat{E}^{(+)}\right| \pm\right\rangle\right|^{2}$, we expect

$$
S\left(\omega_{ \pm}\right) \propto E_{1 / 2}^{2} c^{2}+E_{2 / 1}^{2} s^{2} \pm 2 c s E_{1} E_{2} .
$$

These equations can qualitatively explain the features in Fig. 3(c). Namely, for $\theta=0$, the two dipoles are aligned, so both peaks exhibit the same dependence with the polarizer angle $\varphi$. For $\theta=\pi / 2$, the two are orthogonal, $E_{1} \propto \cos \varphi, E_{2} \propto \sin \varphi$. A frequency-resolved polarization 
measurement therefore provides valuable information about the internal structure of the dimer, since the angle between the two lobes gives an estimate of $\theta$. However, the information obtained about the presence of coherent dynamics is limited: only in the case of aligned dipoles and $\beta=\pi / 2$, the presence of two peaks with the same $\varphi$ dependence unambiguously signifies the absence of coherent coupling. All the other cases shown in Fig. 3(c), are ambiguous, i.e., one cannot tell whether the peaks are created by uncoupled or coupled emitters.

Coherent couplings can, however, be unveiled by correlation measurements in the frequency domain. Without loss of generality, we focus on the case $\theta=\pi / 2$. We analyze cross-correlations between the two emission peaks at $\omega_{ \pm}$ [see Fig. 3(b)], since any possible two-photon deexcitation process from $|d\rangle$ will involve one photon at each of those two frequencies [see Fig. 1(c)]. As we show in Figs. 3(d) and 3(e), measuring this cross-peak correlation for different values of $\varphi$ reveals bunching features $\left[g^{(2)}\left(\omega_{+}, \omega_{-}\right)>1\right]$ only in the presence of coherent coupling, even if the steady-state coherence is zero in all cases. To qualitatively understand this observation, we note that, at $\beta=0$ and $\varphi=\pi / 4$, coherent coupling yields a destructive interference of the emission at $\omega_{-}$originating from the firstexcitation subspace, i.e., $S\left(\omega_{-}\right)=0$ in Eq. (4). Consequently, any emission detected at $\omega_{-}$must originate from the transition from the doubly excited state $|d\rangle$ to state $|+\rangle$, and will therefore show strong correlations with subsequently emitted photons from $|+\rangle$ to $|g g\rangle$ at frequency $\omega_{+}$. The position of bunching feature at $\varphi=\pi / 4$ moves as $\beta$ increases due to the induced rotation of the polarization on the emission lines as $\beta$ varies [see two rightmost figures in panel (c)]. In the absence of coupling, the two peaks must originate from two independent, detuned emitters, and one recovers the expected result for uncorrelated emission, $g^{(2)}\left(\omega_{+}, \omega_{-}\right) \approx 1$ [69]. Note the qualitative difference between the statistics of coupled and uncoupled cases [Fig. 3(e)] as compared to the spectrum [Fig. 3(c)].

Conclusions. - We have established the potential of steady-state photon correlation measurements as a novel tool for molecular spectroscopy. The analysis of the emission statistics of different models of molecular aggregates revealed that it is possible to detect the presence of environment-induced steady state coherence in the system. A detailed analysis of larger systems will be pursued in future research. In frequency-resolved measurements, we observe that, akin to cross-peaks in two-dimensional laser spectroscopy [48], the bunching of photons at two different frequencies indicates coherent coupling between the dipoles. The strength of the coherent coupling can be read off the bunching ratio, and provides direct access to the bare emitter frequencies, whose accurate determination is of critical importance to relate structural and optical properties in molecular aggregates [50-52]. Our results clearly demonstrate that photon correlations can provide a new valuable tool for experimentalists and open new avenues in the field of quantum spectroscopy $[70,71]$.

The authors would like to thank Graham Fleming for helpful discussions. C.S.M. is funded by the Marie Sklodowska-Curie Fellowship QUSON (Project No. 752180). F. S. acknowledges funding from the European Research Council under the European Union's Seventh Framework Programme (FP7/2007-2013) Grant Agreement No. 319286 Q-MAC.

Both authors contributed equally to this project.

[1] S. Vinjanampathy and J. Anders, Quantum thermodynamics, Contemp. Phys. 57, 545 (2016).

[2] Thermodynamics in the Quantum Regime: Fundamental Aspects and New Directions, edited by F. Binder, L. A. Correa, C. Cogolin, J. Anders, and G. Adesso (Springer International Publishing, Cham, 2018).

[3] A. Streltsov, G. Adesso, and M. B. Plenio, Colloquium: Quantum coherence as a resource, Rev. Mod. Phys. 89, 041003 (2017).

[4] M. Lostaglio, K. Korzekwa, D. Jennings, and T. Rudolph, Quantum Coherence, Time-Translation Symmetry, and Thermodynamics, Phys. Rev. X 5, 021001 (2015).

[5] C.-M. Li, N. Lambert, Y.-N. Chen, G.-Y. Chen, and F. Nori, Witnessing quantum coherence: From solid-state to biological systems, Sci. Rep. 2, 885 (2012).

[6] M. Marcus, G. C. Knee, and A. Datta, Towards a spectroscopic protocol for unambiguous detection of quantum coherence in excitonic energy transport, Faraday Discuss. 221, 110 (2020).

[7] M. O. Scully, K. R. Chapin, K. E. Dorfman, M. B. Kim, and A. Svidzinsky, Quantum heat engine power can be increased by noise-induced coherence, Proc. Natl. Acad. Sci. U.S.A. 108, 15097 (2011).

[8] M. T. Mitchison, M. P. Woods, J. Prior, and M. Huber, Coherence-assisted single-shot cooling by quantum absorption refrigerators, New J. Phys. 17, 115013 (2015).

[9] M. Wertnik, A. Chin, F. Nori, and N. Lambert, Optimizing co-operative multi-environment dynamics in a dark-stateenhanced photosynthetic heat engine, J. Chem. Phys. 149, 084112 (2018).

[10] L. Wang, M. A. Allodi, and G. S. Engel, Quantum coherences reveal excited-state dynamics in biophysical systems, Nat. Rev. Chem. 3, 477 (2019).

[11] A. E. Allahverdyan, R. Balian, and T. M. Nieuwenhuizen, Maximal work extraction from finite quantum systems, Europhys. Lett. 67, 565 (2004).

[12] J. Klatzow, J. N. Becker, P. M. Ledingham, C. Weinzetl, K. T. Kaczmarek, D. J. Saunders, J. Nunn, I. A. Walmsley, R. Uzdin, and E. Poem, Experimental Demonstration of Quantum Effects in the Operation of Microscopic Heat Engines, Phys. Rev. Lett. 122, 110601 (2019).

[13] K. E. Dorfman, D. Xu, and J. Cao, Efficiency at maximum power of a laser quantum heat engine enhanced by noiseinduced coherence, Phys. Rev. E 97, 042120 (2018). 
[14] G. D. Scholes, G. R. Fleming, A. Olaya-Castro, and R. van Grondelle, Lessons from nature about solar light harvesting, Nat. Chem. 3, 763 (2011).

[15] E. Romero, V. I. Novoderezhkin, and R. van Grondelle, Quantum design of photosynthesis for bio-inspired solarenergy conversion, Nature (London) 543, 355 (2017).

[16] G. D. Scholes, G. R. Fleming, L. X. Chen, A. AspuruGuzik, A. Buchleitner, D. F. Coker, G. S. Engel, R. van Grondelle, A. Ishizaki, D. M. Jonas, J. S. Lundeen, J. K. McCusker, S. Mukamel, J. P. Ogilvie, A. Olaya-Castro, M. A. Ratner, F. C. Spano, K. B. Whaley, and X. Zhu, Using coherence to enhance function in chemical and biophysical systems, Nature (London) 543, 647 (2017).

[17] S. Mukamel, Principles of Nonlinear Optical Spectroscopy, Oxford Series in Optical and Imaging Sciences (Oxford University Press, Oxford, UK, 1999).

[18] P. Brumer and M. Shapiro, Molecular response in onephoton absorption via natural thermal light vs. pulsed laser excitation, Proc. Natl. Acad. Sci. U.S.A. 109, 19575 (2012).

[19] H.-G. Duan, V. I. Prokhorenko, R. J. Cogdell, K. Ashraf, A. L. Stevens, M. Thorwart, and R. J. D. Miller, Nature does not rely on long-lived electronic quantum coherence for photosynthetic energy transfer, Proc. Natl. Acad. Sci. U.S.A. 114, 8493 (2017).

[20] A. Chenu and P. Brumer, Transform-limited-pulse representation of excitation with natural incoherent light, J. Chem. Phys. 144, 044103 (2016).

[21] H. C. H. Chan, O. E. Gamel, G. R. Fleming, and K. B. Whaley, Single-photon absorption by single photosynthetic light-harvesting complexes, J. Phys. B 51, 054002 (2018).

[22] A. Dodin and P. Brumer, Light-induced processes in nature: Coherences in the establishment of the nonequilibrium steady state in model retinal isomerization, J. Chem. Phys. 150, 184304 (2019).

[23] Y. Fujihashi, R. Shimizu, and A. Ishizaki, Generation of pseudo-sunlight via quantum entangled photons and the interaction with molecules, arXiv:1904.11669.

[24] S. Tomasi, S. Baghbanzadeh, S. Rahimi-Keshari, and I. Kassal, Coherent and controllable enhancement of lightharvesting efficiency, Phys. Rev. A 100, 043411 (2019).

[25] J. Iles-Smith, N. Lambert, and A. Nazir, Environmental dynamics, correlations, and the emergence of noncanonical equilibrium states in open quantum systems, Phys. Rev. A 90, 032114 (2014).

[26] T. V. Tscherbul and P. Brumer, Quantum coherence effects in natural light-induced processes: Cis-trans photoisomerization of model retinal under incoherent excitation, Phys. Chem. Chem. Phys. 17, 30904 (2015).

[27] J. Iles-Smith, A. G. Dijkstra, N. Lambert, and A. Nazir, Energy transfer in structured and unstructured environments: Master equations beyond the Born-Markov approximations, J. Chem. Phys. 144, 044110 (2016).

[28] A. Dodin, T. V. Tscherbul, and P. Brumer, Coherent dynamics of V-type systems driven by time-dependent incoherent radiation, J. Chem. Phys. 145, 244313 (2016).

[29] V. N. Shatokhin, M. Walschaers, F. Schlawin, and A. Buchleitner, Coherence turned on by incoherent light, New J. Phys. 20, 113040 (2018).

[30] T. V. Tscherbul and P. Brumer, Non-equilibrium stationary coherences in photosynthetic energy transfer under weak-field incoherent illumination, J. Chem. Phys. 148, 124114 (2018).

[31] P. Brumer, Shedding (incoherent) light on quantum effects in light-induced biological processes, J. Phys. Chem. Lett. 9, 2946 (2018).

[32] D. M. Rouse, E. M. Gauger, and B. W. Lovett, Optimal power generation using dark states in dimers strongly coupled to their environment, New J. Phys. 21, 063025 (2019).

[33] E. Romero, R. Augulis, V. I. Novoderezhkin, M. Ferretti, J. Thieme, D. Zigmantas, and R. van Grondelle, Quantum coherence in photosynthesis for efficient solar-energy conversion, Nat. Phys. 10, 676 (2014).

[34] F. D. Fuller, J. Pan, A. Gelzinis, V. Butkus, S. S. Senlik, D. E. Wilcox, C. F. Yocum, L. Valkunas, D. Abramavicius, and J. P. Ogilvie, Vibronic coherence in oxygenic photosynthesis, Nat. Chem. 6, 706 (2014).

[35] C. Hettich, C. Schmitt, J. Zitzmann, S. Kühn, I. Gerhardt, and V. Sandoghdar, Nanometer resolution and coherent optical dipole coupling of two individual molecules, Science 298, 385 (2002).

[36] T. P. Krüger, V. I. Novoderezhkin, C. Ilioaia, and R. van Grondelle, Fluorescence spectral dynamics of single LHCII trimers, Biophys. J. 98, 3093 (2010).

[37] R. Hildner, D. Brinks, J. B. Nieder, R. J. Cogdell, and N. F. van Hulst, Quantum coherent energy transfer over varying pathways in single light-harvesting complexes, Science $\mathbf{3 4 0 ,}$ 1448 (2013).

[38] E. Wientjes, J. Renger, A. G. Curto, R. Cogdell, and N. F. van Hulst, Strong antenna-enhanced fluorescence of a single light-harvesting complex shows photon antibunching, Nat. Commun. 5, 4236 (2014).

[39] M. Liebel, C. Toninelli, and N. F. van Hulst, Roomtemperature ultrafast nonlinear spectroscopy of a single molecule, Nat. Photonics 12, 45 (2018).

[40] F. Kyeyune, J. L. Botha, B. van Heerden, P. Malý, R. van Grondelle, M. Diale, and T. P. J. Krüger, Strong plasmonic fluorescence enhancement of individual plant light-harvesting complexes, Nanoscale 11, 15139 (2019).

[41] E. del Valle, A. Gonzalez-Tudela, F. P. Laussy, C. Tejedor, and M. J. Hartmann, Theory of Frequency-Filtered and Time-Resolved N-Photon Correlations, Phys. Rev. Lett. 109, 183601 (2012).

[42] A. Gonzalez-Tudela, F. P. Laussy, C. Tejedor, M. J. Hartmann, and E. del Valle, Two-photon spectra of quantum emitters, New J. Phys. 15, 033036 (2013).

[43] C. Sánchez Muñoz, E. del Valle, C. Tejedor, and F. P. Laussy, Violation of classical inequalities by photon frequency filtering, Phys. Rev. A 90, 052111 (2014).

[44] A. Ulhaq, S. Weiler, S. M. Ulrich, R. Roßbach, M. Jetter, and P. Michler, Cascaded single-photon emission from the Mollow triplet sidebands of a quantum dot, Nat. Photonics 6, 238 (2012).

[45] M. Peiris, B. Petrak, K. Konthasinghe, Y. Yu, Z. C. Niu, and A. Muller, Two-color photon correlations of the light scattered by a quantum dot, Phys. Rev. B 91, 195125 (2015).

[46] B. Silva, C. Sánchez Muñoz, D. Ballarini, A. G. Tudela, M. de Giorgi, G. Gigli, K. W. West, L. Pfeiffer, E. del Valle, 
D. Sanvitto, and F. P. Laussy, The colored Hanbury BrownTwiss effect, Sci. Rep. 6, 37980 (2016).

[47] D. I. H. Holdaway, V. Notararigo, and A. Olaya-Castro, Perturbation approach for computing frequency- and timeresolved photon correlation functions, Phys. Rev. A 98, 063828 (2018).

[48] M. Cho, Coherent two-dimensional optical spectroscopy, Chem. Rev. 108, 1331 (2008).

[49] P. Hamm and M. Zanni, Concepts and Methods of 2D Infrared Spectroscopy (Cambridge University Press, New York, 2011).

[50] J. Adolphs, F. Müh, M. E.-A. Madjet, and T. Renger, Calculation of pigment transition energies in the FMO protein, Photosynth. Res. 95, 197 (2008).

[51] Y.-C. Cheng and G. R. Fleming, Dynamics of light harvesting in photosynthesis, Annu. Rev. Phys. Chem. 60, 241 (2009).

[52] M. T. W. Milder, B. Brüggemann, R. van Grondelle, and J. L. Herek, Revisiting the optical properties of the FMO protein, Photosynth. Res. 104, 257 (2010).

[53] N.S. Ginsberg, Y.-C. Cheng, and G. R. Fleming, Twodimensional electronic spectroscopy of molecular aggregates, Acc. Chem. Res. 42, 1352 (2009).

[54] G. S. Schlau-Cohen, A. Ishizaki, and G. R. Fleming, Twodimensional electronic spectroscopy and photosynthesis: Fundamentals and applications to photosynthetic lightharvesting, Chem. Phys. 386, 1 (2011).

[55] N. H. C. Lewis, N. L. Gruenke, T. A. A. Oliver, M. Ballottari, R. Bassi, and G. R. Fleming, Observation of electronic excitation transfer through light harvesting complex ii using two-dimensional electronic-vibrational spectroscopy, J. Phys. Chem. Lett. 7, 4197 (2016).

[56] K. Müller, A. Rundquist, K. A. Fischer, T. Sarmiento, K. G. Lagoudakis, Y. A. Kelaita, C. Sánchez Muñoz, E. del Valle, F. P. Laussy, and J. Vučković, Coherent generation of nonclassical light on chip via detuned photon blockade, Phys. Rev. Lett. 114, 233601 (2015).

[57] C. Hamsen, K. N. Tolazzi, T. Wilk, and G. Rempe, TwoPhoton Blockade in an Atom-Driven Cavity QED System, Phys. Rev. Lett. 118, 133604 (2017).

[58] R. Tenne, U. Rossman, B. Rephael, Y. Israel, A. KrupinskiPtaszek, R. Lapkiewicz, Y. Silberberg, and D. Oron, Superresolution enhancement by quantum image scanning microscopy, Nat. Photonics 13, 116 (2019).

[59] C. Sánchez Muñoz, B. Buča, J. Tindall, A. GonzálezTudela, D. Jaksch, and D. Porras, Symmetries and conservation laws in quantum trajectories: Dissipative freezing, Phys. Rev. A 100, 042113 (2019).

[60] K. E. Dorfman and S. Mukamel, Multidimensional photon correlation spectroscopy of cavity polaritons, Proc. Natl. Acad. Sci. U.S.A. 115, 1451 (2018).

[61] Z. Zhang, P. Saurabh, K. E. Dorfman, A. Debnath, and S. Mukamel, Monitoring polariton dynamics in the LHCII photosynthetic antenna in a microcavity by two-photon coincidence counting, J. Chem. Phys. 148, 074302 (2018).

[62] P. Kjellberg, B. Brüggemann, and T. Pullerits, Twodimensional electronic spectroscopy of an excitonically coupled dimer, Phys. Rev. B 74, 024303 (2006).

[63] A. V. Pisliakov, T. Mančal, and G. R. Fleming, Twodimensional optical three-pulse photon echo spectroscopy. II. Signatures of coherent electronic motion and exciton population transfer in dimer two-dimensional spectra, J. Chem. Phys. 124, 234505 (2006).

[64] L. Chen, R. Zheng, Q. Shi, and Y. Yan, Two-dimensional electronic spectra from the hierarchical equations of motion method: Application to model dimers, J. Chem. Phys. 132, 024505 (2010).

[65] J. Yuen-Zhou and A. Aspuru-Guzik, Quantum process tomography of excitonic dimers from two-dimensional electronic spectroscopy. I. General theory and application to homodimers, J. Chem. Phys. 134, 134505 (2011).

[66] P. Bhattacharyya and G. R. Fleming, Two-dimensional electronic-vibrational spectroscopy of coupled molecular complexes: A near-analytical approach, J. Phys. Chem. Lett. 10, 2081 (2019).

[67] A. Halpin, P. J. M. Johnson, R. Tempelaar, R. S. Murphy, J. Knoester, T. L. C. Jansen, and R. J. D. Miller, Twodimensional spectroscopy of a molecular dimer unveils the effects of vibronic coupling on exciton coherences, Nat. Chem. 6, 196 (2014).

[68] M. O. Scully and M.S. Zubairy, Quantum Optics (Cambridge University Press, Cambridge, England, 1997).

[69] See Supplemental Material at http://link.aps.org/ supplemental/10.1103/PhysRevLett.124.203601 for further information.

[70] K. E. Dorfman, F. Schlawin, and S. Mukamel, Nonlinear optical signals and spectroscopy with quantum light, Rev. Mod. Phys. 88, 045008 (2016).

[71] F. Schlawin, K. E. Dorfman, and S. Mukamel, Entangled two-photon absorption spectroscopy, Acc. Chem. Res. 51, 2207 (2018). 